\title{
Recognition of Fruit and Vegetables as Healthful: Vitamins and Phytonutrients
}

I.L. Goldman

Summary. Plants are the foundation for a significant part of human medicine and for many of the most widely used drugs designed to prevent, treat, and cure disease. Folkloric information concerning traditional remedies for disease has had inestimable value in establishing familial and cultural linkages. During the $20^{\text {th }}$ century, modern medical science in the U.S. and other developed countries ushered in a new era focused on synthetic medicines. Even though many of these compounds were based on natural compounds found in plants, the drive towards synthetic pharmaceuticals created a knowledge gap concerning the health functionality of plants, crops, and food. Paralleling this development, biochemists and nutritional scientists pioneered the discovery of vitamins during the early decades of the $20^{\text {th }}$ century. This research paved the way for dietary guidelines based on empirical data collected from animal feeding trials and set the stage for the current emphasis on phytonutrients. Three primary stages characterize the use of fruits and vegetable in human health. The first stage concerns the observation that many fruit and vegetable crops were originally domesticated for their medicinal properties. Making their way into the diet for this purpose, fruit and vegetable crops remained on the fringe from a culinary point of view. The second stage began when the role of vitamins became more widely understood, and fruit and vegetable plants were quickly recognized as a rich source of certain vitamins, minerals, and fiber. At this point, they became more than just an afterthought in the diet of most U.S. citizens. Cartoon icons such as Popeye made the case for the health functionality of leafy greens, while parents schooled their children on the virtues of carrots (Daucus carota), broccoli (Brassica olevacea), and green beans (Phaseolus vulgaris). This renaissance resulted in large increases in fresh fruit and vegetable consumption across the country, a trend that continues to this day. The third phase can be characterized by the recognition that fruit and vegetable crops contain compounds that have the potential to influence health beyond nutritional value. These so-called functional foods figure prominently in the dietary recommendations developed during the last decades of the $20^{\text {th }}$ century. In recent years, surveys suggest nearly two-thirds of grocery shoppers purchase food specifically to reduce the risk of, or manage a specific health condition. Evidence abounds that consumers, including Baby Boomers, choose foods for specific health benefits, such as the antioxidant potential of vegetables, suggesting high levels of nutritional literacy. Clinical and in vitro data have, to some degree, supported the claims that certain foods have the potential to deter disease, however much research remains to be conducted in order to definitively answer specific dietary-based questions about food and health.

The term vegetable is problematic from a scientific point of view. Most people have a strong sense of what constitutes a vegetable and this is often based on its culinary usage. Vegetables are best characterized by their high moisture content and immaturity from a developmental standpoint, but we lack a comprehensive scientific term for this group of edible plants. In part for this reason, communication about vegetable plants can be confusing and misleading. Although a number of exceptions exist, I refer to vegetable crops as those edible, high-moisture content, immature plants or plant parts. Much of the uniqueness of vegetable crops stems from this high moisture content, which dictates special care in harvest, handling, postharvest storage, and culinary preparations.

Associate Professor, Department of Horticulture, University of Wisconsin-Madison, 1575 Linden Drive, Madison, WI 53706; e-mail ilgoldma@facstaff.wisc.edu.

I thank B. Wolfgang Hoffman of the University of Wisconsin College of Agricultural and Life Sciences for his photographic expertise in producing Fig. 3. 
Many vegetable crops have been used for medicinal purposes in addition to their use as food for thousands of years (Janick, 2003). In some cases, it is possible that vegetable crops were domesticated for this reason (Rubatzky and Yamaguchi, 1997; Smartt and Simmonds, 1995), although information of this kind is not widely available. Herbals testify to the plethora of healthrelated properties that have been ascribed to vegetable crops throughout the past several hundred years (Janick, 2003), many of which appear to be connected to the first written records about these crops. Tomato (Lycopersicon esculentum) was used for the treatment of eye diseases and scabies and lettuce (Lactuca sativa) was used medicinally for many centuries before its use as a modern salad crop (Rubatzky and Yamaguchi, 1997). The alkaloids present in tomato and latex present in lettuce were assigned responsibility for these medicinal properties. Many similar relationships between secondary compounds from vegetable crops and human health have been cited, including the myriad of health-related properties associated with vegetable alliums, such as onion (Allium cepa) and garlic (Allium sativum) (Block, 1992). For example, the frequent use of garlic by Babylonians, Egyptians, Phoenicians, Vikings, Chinese, Greeks, Romans, and Hindus makesitone of the most widely used vegetables in folkloric medicine (Amagase et al., 2001). Today, as our interest in the relationship between crops and human health grows, it should not be surprising to us to learn that many of our staple vegetable crops were used as medicinals, and perhaps simultaneously as food crops.

Interestingly, many of the medicinal properties described in folklore and, to some extent, verified by modern medical research have their origins as plant protectants (Block, 1992). Secondary compounds are often associated with defensive functions in plants, and many of these compounds are of great interest today as phytonutrients. Compounds such as flavonoids, carotenoids, terpenes, glucosinolates, isoflavones, and thiosulfinates may, through their inherent toxicity, confer significant opportunities for pest control (Drewnoski and Gomez-Carneros, 2000). However, this astringency and toxicity that may be important for survival is viewed widely be consumers as negative from a culinary point of view. Thus, agricultural practices, including plant breeding and postharvest handling, have attempted to reduce or minimize the astringency of secondary compounds that impact vegetable flavor (Drewnoski and Gomez-Carneros, 2000). The vegetable crops of today may therefore be modified significantly from their earlier domesticated counterparts with respect to such phytonutrients. For this reason, it is possible that consumers today do not see the close linkage between crops and human health that may have been apparent to human societies living more closely to the period of transition between hunter-gatherers and agriculture.

\section{Perceptions of vegetables in the U.S.}

Vegetable crops were widely cultivated by native Americans for perhaps thousands of years before the arrival of European immigrants in the early $17^{\text {th }}$ century. Vegetables were also a staple of the immigrant Puritans and Pilgrims, who settled the territories that later became the U.S. These pioneers brought vegetable seeds with them on their voyages and used these seeds to develop and select vegetable crops suited to life on the Atlantic coast (Goldman et al., 2000). As the population of the U.S. moved westward, vegetable crops remained important components of the diet. In The Homestead, a 1936 painting by the American Regionalist painter John Steuart Curry, depicting pioneer life on the western plains, cabbage (Brassica oleracea) is cultivated in a kitchen garden, alongside the grains and forages that made up the basis of the diet for the farm family and its livestock (Fig. 1) (Junker, 1998). Vegetables provided diversity in the farm diet and also delivered the promise of cures for a variety of ailments, passed-down through the generations in an oral tradition that bound families and cultures together. The kitchen garden has been a mainstay of rural life in America for at least several centuries (Darlington and Moll, 1907). Its emphasis on vegetable crops pro-

Fig. 1. The Homestead. 1936. John Steuart Curry (from Junker, 1998).

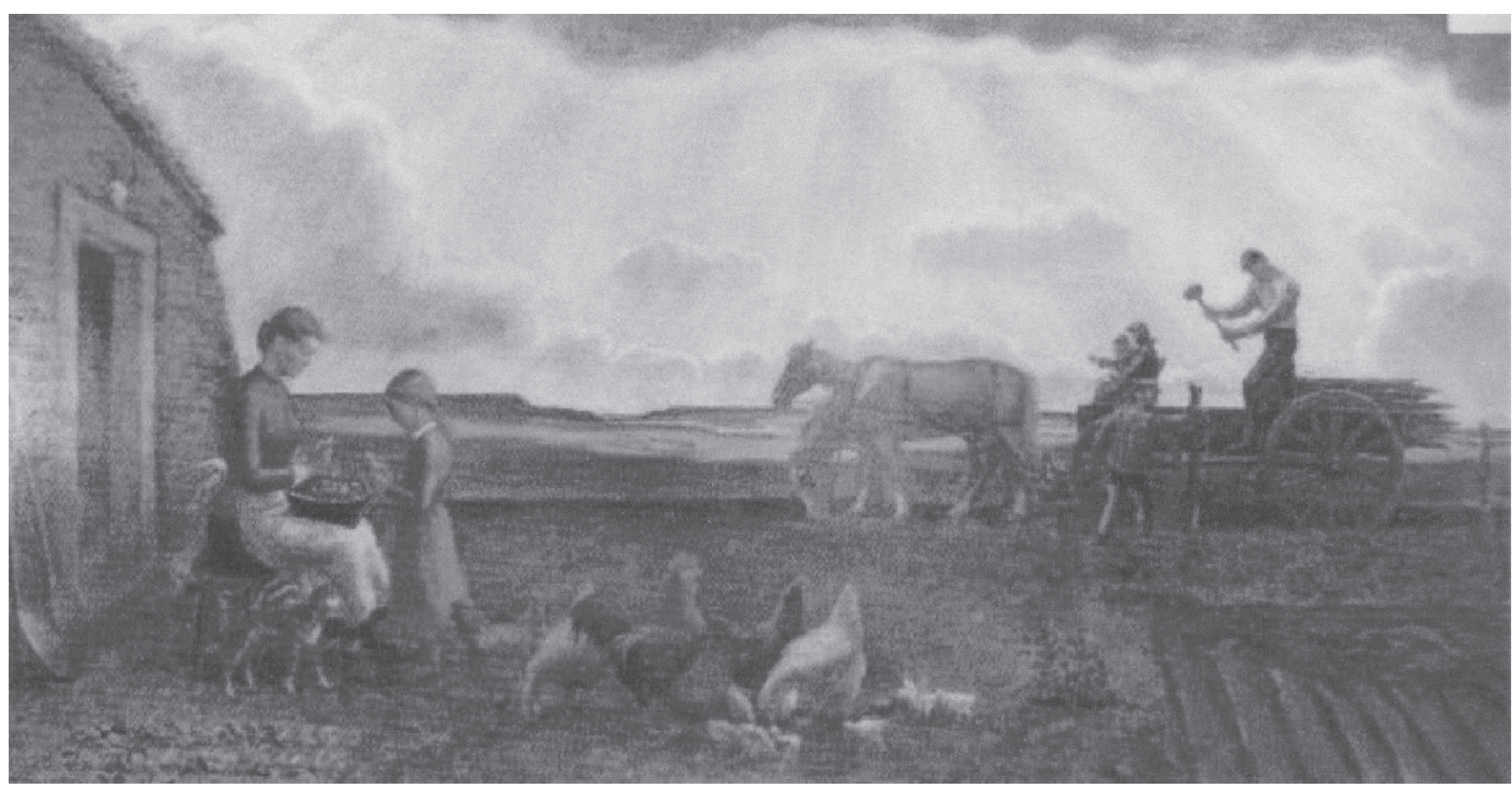


vided the continuity of vegetable cultivation and use between Europe and the U.S., and also perhaps served as a cultural bridge between European and American life.

As the traditions of vegetable cultivation stayed strong in many parts of the U.S., people still relied heavily on vegetables and herbs for their healing properties. However, a marked shift away from these practices and toward synthetic medicine took place during the $20^{\text {th }}$ century (Lawson, 1998). This trend was particularly acute as modern, synthetic pharmaceuticals were developed through advanced chemical means. These synthetic monomolecular drugs became a focal point for the practice of modern medicine in the U.S. and many other parts of the developed world, leading to tremendous gains in public health. However, with the shift away from plant-based remedies came a loss of knowledge about their uses and efficacy. While this knowledge was retained and even strengthened in Europe, it tended to disappear in the U.S. to the point that generations born following World War II were far less inclined to turn toward food-based remedies for health concerns, and instead focused on the more widely-available and highly efficacious monomolecular drugs (Lawson, 1998).

Nutritional science has gone through several distinct phases, among them 1) a naturalistic era, in which mystical cures were attributed to plantbased compounds and whole foods; 2 ) a chemical-analytical era, in which the discovery of atoms and molecules that comprise food led to analysis of the components of food from a chemical point of view; and 3 ) the biological era, which began in the early part of the $20^{\text {th }}$ century and led to the recognition of specific elements of food and their functional properties, such as the discovery of vitamins (McCollum, 1957; University of Vermont, 2001). In what might appear a throwback to the naturalistic era, we have again become interested in the healing properties of foods. While not eschewing the insight of the chemical-analytical and biological eras, we seek new knowledge about the connection between crops and human health to supplement our understanding of components of these plants identified in the previous eras. Furthermore, the vegetable crops are among the crop groups with the greatest human health interest, due in large part to their close connec- tion with medicinal properties stemming from domestication, and the large body of folkloric information about medicinal uses spanning virtually all of the world's human cultures.

In the past decade, the scientific field that combines knowledge about crops and human health has come to be known as functional foods. Functional foods are so-called because they deliver some physiological benefit beyond nutrition. An excellent example of a functional food is carrot (Daucus carota), which contains carotenoids that deliver antioxidant activity in addition to their pro-vitamin A activity. This multi-functionality of the molecule renders the carrot, and possibly other foods containing carotenoids, as functional for health. Many products in today's marketplace are labeled for health functionality, and the limits of these claims are currently being tested at both the regulatory agency and clinical research levels (Sloan, 2000).

Given this broad definition, it is certainly possible that all foods are functional. Perhaps we have simply not discovered the functionality of various molecules, themselves possibly undiscovered, in foods that are commonplace in our diet. Alternatively, many of these secondary compounds possess toxic effects for the purpose of pest control, as discussed above. Toxicity may contribute to functionality, as in the case of eliminating unwanted cells or it may limit functional abilities, in the case of adding toxins. The science of food functionality is therefore complex and likely includes both positive and negative effects of secondary compounds from a single food source. Finally, although it is clear that these functional molecules provide additional physiological benefits to the consumer beyond their basic nutritional value, they may represent a very small slice of potentially functional and anti-functional molecules whose actions remain unknown at the present time.

\section{Birth of food functionality}

Before the first decade of the $20^{\text {th }}$ century, nutritional scientists broke food into several simple categories. This often included carbohydrate or starch, protein, fat, and mineral components, but little else, as vitamins had yet to be discovered (Welsh et al., 1992). As modern methods of science began to take hold in the early part of the $20^{\text {th }}$ century, nutritional scientists ap- proached dietary constituents with a critical eye. As they began to focus on the question of what kinds and amounts of specific nutritional components are required for human and animal health, experimental systems were devised to examine dietary guidelines. These systems proved an important beginning for our perspectives on the health-functionality of food.

The pioneering work of E.V. McCollum, a USDA employee at the University ofWisconsin, laid the groundwork for the discovery of vitamins. McCollum made several important decisions in his research program that allowed for these discoveries to take place. He was among the first to use rats (Rattusnorvegious) instead of cows (Bos tarus) for experimental nutrition research, thereby greatly reducing the time and cost associated with these kinds of experiments and increasing the potential for discovery. It is well known that the development of model organisms has fueled the growth of many fields of science, and nutritional science was no exception. The purified diets McCollum used in his rat studies differed in only a single constituent, thereby allowing for more precise interpretations of experimental data. This method came to be known as the method of biological analysis, which allowed for more comprehensive and realistic analysis of food and its components in the animal diet than previous methods that focused solely on chemical composition. By feeding rats purified diets, McCollum was able to more carefully and unambiguously identify the important constituents of foods for growth and sustained health (McCollum 1957).

McCollum was the first to identify an important fat-soluble constituent of food from butterfat and egg yolk, which later became known as Vitamin A. At the time of this discovery, McCollum wrote that there appeared to be evidence for "certain accessory articles in certain food-stuffs which are essential for normal growth for extended periods." The word vitamin was coined by Casimir Funk in 1912. Later, McCollum and his research group identified a water-soluble element known as Vitamin B. McCollum's work was aided greatly by the collaborative efforts of $\mathrm{H}$. Steenbock, a biochemist at the University of Wisconsin. Steenbock's confirmation of McCollum's work, along with his discovery that irradiation of food produced vitamin $\mathrm{D}$, led to many im- 
portant advances in the health-functionality of food. In addition, patents covering these discoveries were important contributors, through endowments established with the Wisconsin Alumni Research Foundation, to research in a wide variety of fields at the University of Wisconsin. McCollum moved to the School of Hygiene and Public Health at Johns Hopkins in 1917, where his work led to the discovery of Vitamin $\mathrm{D}$, at that time known as the anti-ricketic vitamin (McCollum, 1957).

Upon reflection and investigation, important diseases such as pellagra, rickets, and scurvy that had plagued humans for centuries could be attributed to the deficiency of particular vitamins in the diet. While it had been known since perhaps the $17^{\text {th }}$ century that a disease like scurvy could be caused the poor diet and cured by the addition of

A

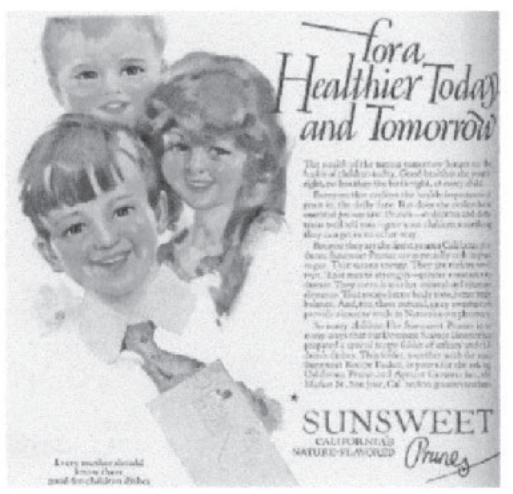

citrus fruit (hence the British sailor's designation as limeys for their consumption of these fruit on long voyages), the specific constituent was not isolated until after McCollum's early work on vitamins A and B. By the 1920s, the specific cause of pellagra, rickets, and scurvy were attributed to vitamin deficiencies, and new support was found for the health-functionality of food.

These pioneering studies led to the discovery of many vitamins, opening up a new area of research in human nutrition. The presence of vitamins in vegetables also cleared the way for more concrete recommendations on the nutritional impact of these foods. It also provided parents with a solid rationale for encouraging their children to eat a more balanced diet. Cartoon icons such as Popeye the Sailor Man made a strong case for vegetable consumption in the
U.S. public. Vegetable marketing efforts were constructed with vitamin content in mind, thereby promoting a science-based value-added commodity to the public.

By the early 1920s, marketers had already begun to take advantage of this new knowledge about food and health. In this period, advertisements start to indicate the emphasis on food functionality for health (Fig. 2). In 1922, The Sunsweet Company, a large seller of dried fruit, advertised prunes in Good Housekeeping as containing both iron and vitamins. The advertisement clearly states that this food is associated "with a healthier today and tomorrow," in many ways similar to advertisements for such products found in today's marketplace (Fig. 2a). An emphasis on vitamin content also indicates that Sunsweet felt consumers could be encouraged to pur-
B

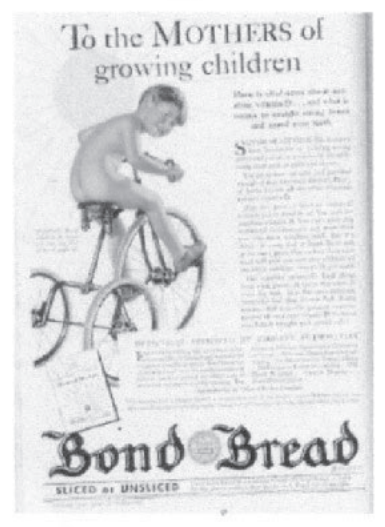

D

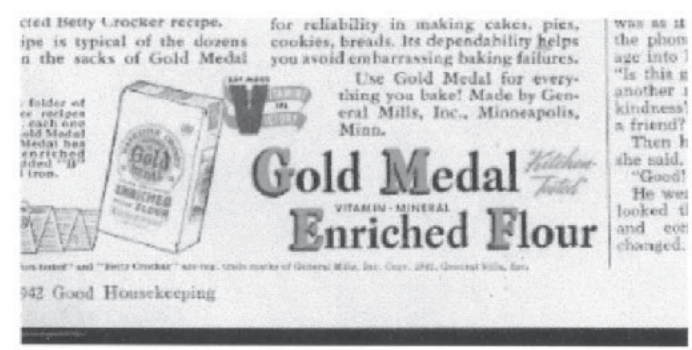

Fig. 2. Advertisements from Good Housekeeping. (a) Sunsweet Prunes, 1922; (b) Bond Bread, 1932; (c) Libby's Vegetables, 1932; (d) Gold Medal Flour, 1942; (e) Sunkist Orange Juice, 1942.

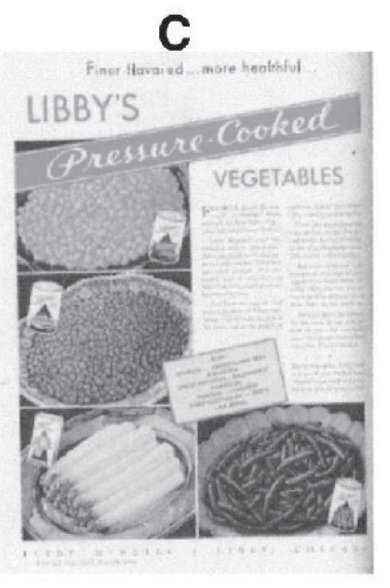

E

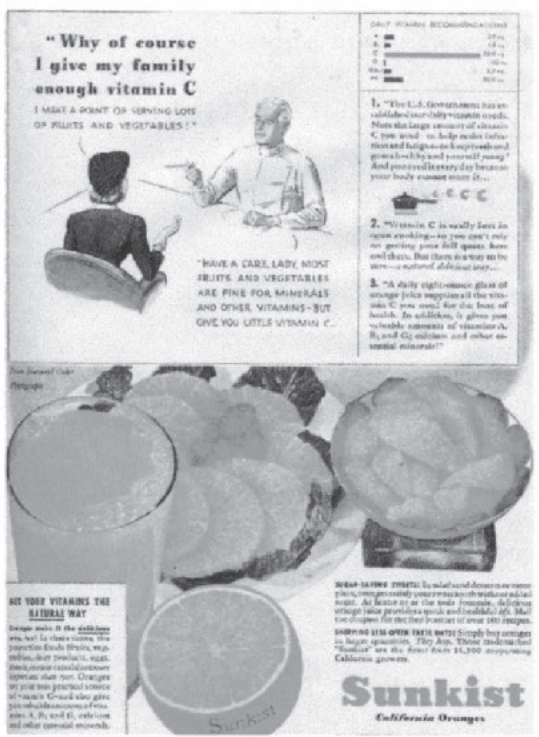


chase their product because the presence of vitamins was highlighted. By 1932, marketers were capitalizing on the public's solid foundation of knowledge about vitamin content. An advertisement for Bond Bread, also found in Good Housekeeping, steers potential customers toward its product because of its value-added vitamin $D$ enrichment. The ad shows a naked boy on a bicycle, absorbing the rays of the sun on his skin (Fig. 2b). The ad asks whether mothers should expect their growing children to obtain their vitamin $\mathrm{D}$ this way or if simply consuming a vitamin-enriched bread would be easier. At this point, it is clear that the technologies associated with enhancing the vitamin content of foods were viewed widely as tremendous public health successes (McCollum 1957). This era also was associated with improvements in canning and other processing technologies, bringing canned and other processed vegetables to a wider range of the U.S. public. As knowledge about the vitamin composition of vegetables grew, canned and other processed vegetable products were marketed for their vitamin composition (Fig. 2c).

During World War II, when domestic agricultural production was needed for support of our troops overseas, fruit and vegetables were in short supply. For this reason, the U.S. government promoted the growing of Victory Gardens by individual citizens (Burdett, 1943). The Victory Garden was an attempt to encourage individuals to cultivate their own land, be it an urban backyard garden or a rural plot of land, to enhance their supply of fresh produce during the war. These efforts were extremely successful in many ways, not the least of which was to reacquaint urban dwellers with the simple idea of growing their own food. It is likely that the opportunity to engage in vegetable production increased awareness of vegetable crops and had an impact on vegetable consumption patterns following the war. A generation of young people growing up in urban centers during World War II had an opportunity to participate directly in the cultivation of vegetable crops, which would likely not have taken place were it not for the war and the Victory Garden program. This was also a period where patriotic slogans were in vogue, including the Vitamins for Victory that can be found on packages of processed flour (Fig. 2d).

Following World War II, the U.S. government made efforts to improve children's nutrition through the $\mathrm{Na}$ tional School Lunch Program, a federal program that is still in existence today. The National School Lunch Program made it possible for children to obtain meals at school that were planned to emphasize proper nutrition. Thus, educators, nutritionists, and parents collaborated on a nutrition program aimed at improving the nutritional status of U.S. children. To authorize funds for this program, the Agricultural Act of 1935 was amended by the $78^{\text {th }}$ congress in July, 1943 (Gunderson, 2001). This provided funds not in excess of $\$ 50$ million for maintaining school lunch and milk programs over the period of one year. Cash payments were made to school lunch sponsors for the purchase of food for the program. The program expanded rapidly in the first few years, and by 1946 some 6.7 million children were beneficiaries of this legislation (Gunderson, 2001). At this time, the National School Lunch Program was made a permanent program in the form of the National School Lunch Act. In Section 2 of this act, the phrase "encourage the domestic consumption of nutritious agricultural commodities" can be found, indicating that there was an emphasis in this program on nutritional composition.

These efforts had the effect of raising awareness about vitamin content of foods, particularly fruits and vegetables, and was important in promoting vegetables to children. Promotional efforts for fruit and vegetable products took on scientific pronouncements, particularly in suggesting that vitamin intake was important for children (Fig. 2e). From this period, nutritional guidelines, such as Recommended Daily Allowances, have been a mainstay of meal planning efforts at state and federal levels. These efforts have their roots in the activities of the Food and Nutrition Board of the National Research Council, which was organized to direct government activities for the wartime nutrition programs (National Academy of Sciences, 1989). As this board reviewed research on requirements for various nutrients, they developed the first set of recommendations, known as the recommended daily allowances (RDAs) in 1943.

\section{Vitamins, vegetables, and the land grant idea}

Throughout the $20^{\text {th }}$ century, the land grant institutions played a major role in elucidating the healthful properties associated with vegetable crops. The land grant schools, founded after the Morrill Act of 1862, and the Agricultural Experiment Stations, founded by the Hatch act of 1887, paved the way for a unique form of interdisciplinary collaboration between agricultural, medical, and nutritional scientists. McCollum and Steenbock's early work on vitamin discovery took place in an environment where studies of domestic animals were in close physical proximity to biochemical and medical research. In such an environment, questions raised about how scientific fields overlap are possible to address. In fact, a culture of interdisciplinary collaboration between scientists encourages such interaction, and in this way many of the early questions about the specific components of food associated with human health were answered.

The campus of the University of Wisconsin in Madison has a long history of such collaborative work that led to vitamin discoveries and applications. These successes have made a large impact on the scientific culture of the institution, particularly as they have been studied and promoted throughout the years by scientists, students, and administrators. In 1936, the Dean of the College of Agricultural and Life Sciences, Chris Christiansen, appointed the American regionalist painter John Steuart Curry as Artist in Residence, a very pioneering sort of appointment for an agricultural school. Curry, a Kansas native, was given the charge of inspiring students and the general public about agriculture, and he chose many of the scientific successes during the early part of the $20^{\text {th }}$ century as material for his canvases. Curry's studio was located in a house on Linden Drive, right in the middle of the agricultural buildings on the campus. In addition to his many works extolling the successes of U.S. agriculture and the relationship between these successes and the land grant institutions, Curry's famous 1942 mural on an interior wall in the Biochemistry building, The Social Benefits of Biochemical Research, depicted the power of these institutions in solving basic agricultural and human nutrition problems through research. In this mural, whitecoated scientists are shown leading children with rickets and other nutritional deficiencies from a darkened farmstead into the light of day, improving their health through the kind of research that 


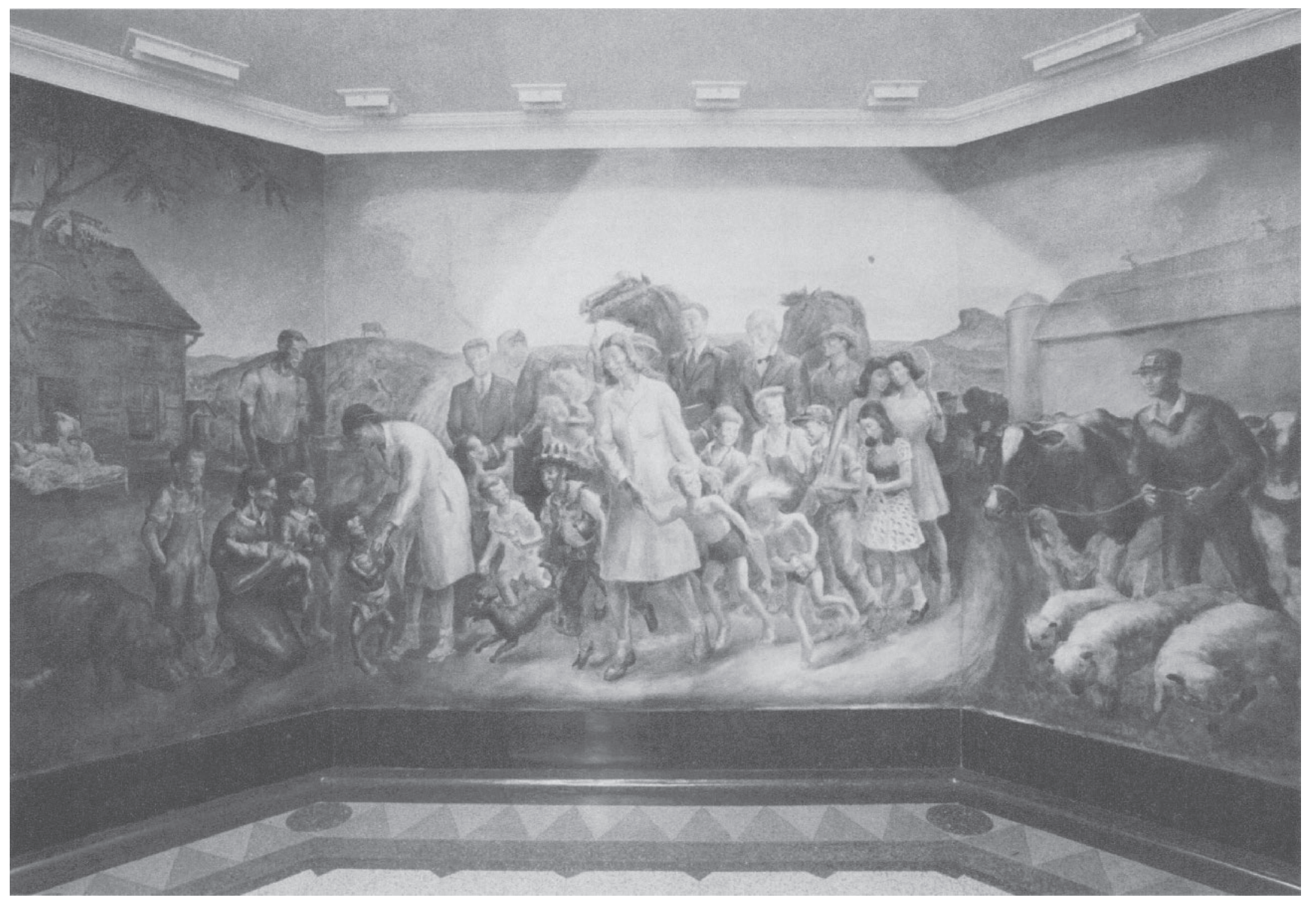

Fig. 3. The Social Benefits of Biochemical Research. 1942. John Steuart Curry (from Junker, 1998).

has characterized the successful land grant institutions (Fig. 3). The mural depicts the challenges pioneer farmers faced in raising livestock as well as the appearance of nutritional disorders in humans that could not be described until the early part of the $20^{\text {th }}$ century. Curry's work stands as a testament to the interdisciplinary nature of research at the land grant institutions and the solution of many basic problems in human and animal nutrition. Clearly, the message Curry was communicating to the students of the campus and to the general public was that science offers a unique opportunity for healing agricultural woes, and the vitamin discoveries were an ideal framework for this artistic expression.

\section{Vegetables and health: Perspectives in the $21^{\text {st }}$ Century}

As nutritional supplementation and nutritional labeling became commonplace in the decades following World War II, consumers were relatively wellinformed about the value of vegetable crops as sources of vitamins and, to some extent, phytonutrients. Recognition of the value of carrot and other vegetable-derived carotenoids such as beta-carotene to human health, particularly for membrane function and proper eyesight, were well documented and widely known during this period. Synthetic vitamins were also developed and sold widely during the 1950s. Consumers were quite aware of both dietary and non-dietary sources of vitamins and minerals and their health functionality, as more information about the specific action of these compounds was being collected, disseminated, and advertised.

During this period, even as agricultural production was registering record levels, large demographic shifts were occurring in the U.S. As more and more people moved to urban centers and away from rural areas, our close connection with the land and with agricultural production was eroded. The political base for agricultural policy in the rural areas began to shrink, and with it came a concomitant increase in public awareness of environmental quality issues associated with agriculture. Publication of Rachel Carson's Silent Spring (Carson, 1962), the introduction of federal legis- lation in the form of the Clean Air and Clean Water Acts in the 1970s, and a large-scale move toward larger, more vertically-integrated farming operations, have all been cited as influential trends in U.S. agricultural history, paving the way for the environmental movements of the 1960s and 1970s. These events signaled a changing relationship between farmers and consumers, who now began to travel in different philosophical directions. In addition, as agricultural production boomed and commodity prices remained low, focus shifted away from simply producing enough and into many important quality factors. Among these quality factors for vegetable crops are color, flavor, nutritional composition, and health-functionality. Because many of the secondary compounds with health-functionality are associated with color (polyphenols) and flavor (isothiocyanates and organosulfur compounds), an emphasis on the interconnected web of quality factors had the effect of focusing on both improved culinary and health factors.

With these large-scale changes in perspective, the relationship between the U.S. consumer and health-related 
aspects of vegetable crops has changed as well. Today, this relationship is characterized by a desire to deliver significant health-functionality to the consuming public through the development and enhancement of functional foods. Some have argued that this trend has been fueled in part by the aging of the baby-boom generation and their search for improving physical and mental health and retarding the aging process. Fortification of food with vitamins and minerals may have signaled the first step in this trend, but today's consumers appear to be interested in food that will serve many purposes, including multiple health-functionalities. Breakfast cereals may contain a multi-vitamin supplement that delivers at least $100 \%$ of the RDA for many vitamins and minerals. Certain brands of pet food contain similar multivitamin supplements and antioxidants. Many brands of orange juice contain calcium, greater than $100 \%$ of the RDA for vitamin C, and $100 \%$ of the RDA for vitamin $\mathrm{E}$, the latter of which are promoted for their antioxidant potential. Carrots with elevated levels of carotenoids have been released by federal and state breeding programs at the University of Wisconsin (Simon, 1997). A great deal of interest has been generated by Beta-Sweet, a purple and orange carrot developed by L. Pike at Texas A\&M University, for its levels of both beta-carotene and anthocyanin (Brown, 2000). Similar largescale public interest has been generated by broccoli sprouts (Brassica oleracea), developed by researchers at Johns Hopkins University, because of their elevated levels of phase II inducing enzymes that have been implicated in anticancer activity (Talalay and Fahey, 2001). Consumer interest in enhancing the multi-functionality of vegetable crops is high, and research is following suit.

The statement attributed to Hippocrates: "Let your food be your medicine and your medicine be your food" certainly applies to today's current emphasis on functional foods. However, it may also be appropriate to ask whether we are expecting too much from our foods for them to solve our physical and mental ailments while providing us with both beauty and sustenance. Despite this lofty expectation, vegetable crops may be one of the few commodities in the functional foods arena that have the potential to do all of these things, as it is clear that they have long been associated with these qualities in various ways since the beginning of agriculture.

Given that many vegetable crops were originally domesticated with medicinal purposes in mind, and that they have been used as both food and medicine by cultures worldwide for millennia, it is not surprising that there exists a resurgence in interest in the close linkage between food and health. Coupled with the aging of a large segment of the U.S. population, the focus on quality factors in vegetable crop production, and the growing body of knowledge in nutrition on the action of specific elements of food, we should expect increased expectations of the health functionality of vegetable crops in future years.

\section{Literature cited}

Amagase, H., B.L. Petesch, H. Matsuura, S. Kasuga, and Y. Itakura. 2001. Intake of garlic and its bioactive components. J. Nutr. 955s-962s.

Block, E. 1992. The organosulfur chemistry of the genus Allium. Implications for organic sulfur chemistry. Angewandte Chemie Intl. Ed. 31:1135-1178.

Brown, K.S. 2000. Food with attitude. Discover 21(3). 11 Nov. 2002. <http:// www.discover.com/mar_00/featfuturetech. html>.

Burdett, J.J. 1943. Victory garden manual. Ziff-Davis, Chicago.

Carson, R. 1962. Silent spring. Fawcett, Greenwich, Conn.

Darlington, E.D. and L.M. Moll. 1907. How and what to grow in a kitchen garden of one acre. E. Atlee Burpee (ed.). W.A. Burpee and Co., Philadelphia.

Drewnoski, A. and C. Gomez-Carneros.
2000. Bitter taste, phytonutrients, and the consumer: A review. Amer. J. Clinical Nutr. 72:1424-1435.

Goldman, I.L., G. Schroeck, and M.J. Havey. 2000. History of public onion breeding programs and pedigree of public onion germplasm releases in the United States. Plant Breeding Rev. 20:67-103.

Gunderson, G.W. 2001. The national school lunch progam. 11 Nov. 2002. <http:// www.fns.usda.gov/cnd/Lunch/default. htm>.

Janick, J. 2003. Herbals: The connection between horticulture and medicine. HortTechnology 13(2):229-238.

Junker, P. 1998. John Steuart Curry: Inventing the middle west. Hudson Mills Press, New York.

Lawson, L.D. 1998. Garlic: A review of its medicinal effects and indicated active compounds, p. 176-209. In: L.D. Lawson and R. Bauer (eds.). Phytomedicines of Europe. Amer. Chem. Soc. Symp. Ser. 691.

McCollum, E. 1957. A history of nutrition: the sequences of ideas in nutrition investigations. Houghton Mifflin, Boston.

National Academy of Sciences, 1989. Food and nutrition board: Recommended daily allowances. $10^{\text {th }}$ ed. Natl. Res. Council, Wash. D.C.

Rubatzky, V.E. and M. Yamaguchi. 1997. World vegetables. $2^{\text {nd }}$ ed. Chapman and Hall, New York.

Simon, P.W. 1997. Plant pigments for color and nutrition. HortScience 32:12-13.

Sloan, A.E. 2000. The top ten functional food trends. Food Technol. 54:1-17.

Smartt, J. and N.W. Simmonds. 1995. Evolution of crop plants. $2^{\text {nd }}$ ed. Longman, New York.

Talalay, P. and J.W. Fahey. 2001. Phytochemicals from cruciferous plants protect against cancer by modulating carcinogen metabolism. J. Nutr. 131:3027s-3033s.

University of Vermont, 2001. A brief history of nutrition. 11 Nov. 2002. <http:// asci.uvm.edu/course/webcourses.htm>.

Welsh, S., C. Davis, and A. Shaw. 1992. A brief history of food guides in the United States. Nutr. Today. Nov./Dec.:6-11. 